\title{
Using global neutron monitor network data for GLE analysis: recent results
}

\author{
Alexander Mishev* \\ Space Climate Research Unit, University of Oulu, Finland. \\ E-mail: alexander.mishev@oulu.fi \\ Ilya Usoskin \\ Space Climate Research Unit; Sodankylä Geophysical Observatory (Oulu unit), University of \\ Oulu, Finland. \\ E-mail: ilya.usoskin@oulu.fi
}

\section{Leon Kocharov}

Sodankylä Geophysical Observatory (Oulu unit), University of Oulu, Finland.

E-mail: leon.kocharov@oulu.fi

The solar cycle 23 provided several strong ground level enhancements (GLEs). The first strong GLE event of the cycle was observed on 14 July 2000 (the Bastille day event), while the last was observed on 13 December 2006. In addition, the period of late October - early November 2003 was characterized by strong cosmic ray variability and sequence of three GLEs. Cycle 23 produced also the second largest event in the observational history - 20 January 2005 event. Here we perform a precise analysis of spectral and angular characteristics of solar energetic particle (SEP) events on the basis of neutron monitor (NM) data. We model particle propagation in the Earth's magnetosphere and atmosphere using a newly computed NM yield function at several altitudes above the sea level. The method includes several consecutive steps: detailed computation of asymptotic cones and rigidity cut-off of each NM station used in the analysis, making an initial guess of the inverse problem by assuming the apparent source position location in a convenient way, application of the NM yield function for detector response modelling and subsequent optimization procedure in order to derive spectral and angular characteristics of SEPs. Here, we present results from analysis of several GLEs. The SEP spectra and pitch angle distributions were obtained in their dynamical development throughout the events. We briefly demonstrate the capability of the method to assess SEP features of sub-GLE events, the details reported in this volum. Several case examples are discussed.

The 35th International Cosmic Ray Conference,

12 - 20 July, 2017

Busan, Korea Republic

\footnotetext{
${ }^{*}$ Speaker.
} 


\section{Introduction}

Thu study of solar energetic particle (SEP) events is important in order to understand the particle acceleration at the Sun as well as their transport in the interplanetary medium $[1,2,3]$. Solar flares and coronal mass ejections (CMEs) accelerate protons as well as ions to high energies $[4,5,6]$. The probability of such events is greater during maximum and decline phase of the solar activity cycle [7]. Occasionally the energy of SEPs is enough high $(\approx 1 \mathrm{GeV} /$ nucleon $)$ to initiate an atmospheric cascade leading to an enhancement of count rate of ground based detectors, specifically NMs. This special class of SEP events is called ground level enhancements (GLEs).

Different methods and instruments have been used to study GLEs events, specifically based on NMs [8, 9, 10, 11]. The worldwide NM network [12], is used as a giant spectrometer [13]. Measurements from NM stations separated in both longitude and latitude covering wide range of rigidity cut-offs are used to derive the spectral characteristics and anisotropy of SEPs by modelling the global NM response and performing a convenient optimization over a space of unknown parameters. In addition, it was recently shown that spectral and angular characteristics of SEPs derived using NM data are model dependent [14]. Therefore, it is very important to analyse GLE events using the same and/or similar models and methods.

\section{Method for derivation of strong SEPs spectra and angular characteristics using global NM network}

The spectral and angular characteristics of SEPs using NM data can be derived using the relationship between the detector count rates and the primary particle flux via the NM yield function, which considers the full complexity of particle transport in the geomagnetosphere and in the Earth atmosphere and the detector response i.e. registration efficiency and effective area itself. The analysis of a GLEs using NM data consists of several consecutive steps: computation of asymptotic viewing cones and rigidity cut-offs of the NMs; making an initial guess of the inverse problem; application of an optimization procedure (inverse method) for derivation of the primary SEPs energy spectrum, anisotropy axis direction, pitch angle distribution. The detailed description of the method is given elsewhere [15]. The method is similar to that used by [10,11]. The relative count rate increase of a given NM is expressed as:

$$
\frac{\Delta N\left(P_{\text {cut }}\right)}{N}=\frac{\int_{P_{\text {cut }}}^{P_{\text {max }}} J_{\| s e p}(P, t) Y(P) G(\alpha(P, t)) A(P) d P}{\int_{P_{\text {cut }}}^{\infty} J_{G C R}(P, t) Y(P) d P}
$$

where $J_{\| \text {sep }}$ is the rigidity spectrum of the primary SEP in the direction of the maximal flux, $J_{G C R}(P, t)$ is the rigidity spectrum of GCR at given time $t$ with the corresponding modulation, $\mathrm{Y}(\mathrm{P})$ is the NM yield function, $G(\alpha(P, t))$ is the pitch angle distribution (PAD) of SEPs, the pitch angle $\alpha$ is defined as the angle between charged particle's velocity vector $\overrightarrow{\mathscr{A}}$ and the direction of the local magnetic field direction $\overrightarrow{\mathscr{C}}$ i.e. $\cos (\alpha)=\overrightarrow{\mathscr{C}} \cdot \overrightarrow{\mathscr{A}}, \mathrm{A}(\mathrm{P})$ is a discrete function with $\mathrm{A}(\mathrm{P})=1$ for allowed trajectory (proton with rigidity $\mathrm{P}$ can reach the station), accordingly $\mathrm{A}(\mathrm{R})=0$ for forbidden trajectory (proton with rigidity $\mathrm{P}$ can't reach the station), the function $A$ is determined during asymptotic cone calculations, $N$ is the count rate due to GCR, $\Delta N\left(P_{\text {cut }}\right)$ is the count rate increase due to solar particles, $P_{\text {cut }}$ is the minimum rigidity cut-off of the station, accordingly $P_{\max }$ is the maximum 
rigidity of SEPs considered in the model, assumed to be $20 \mathrm{GV}$, which is sufficiently high for SEPs. The fractional increase of the count rate of a NM station represents the ratio between the NM count rates due to SEPs and GCR averaged over 2 hours before the event's onset. Here we use a newly computed NM yield function, which provides good agreement with experimental latitude surveys as well as other measurements $[16,17]$. In addition in order to reduce and eliminate uncertainties as the application of two attenuation lengths method i.e. normalization of high altitude NM count rates to the sea level, we employ NM yield functions for different altitudes, i.e. the response of each NM is modelled with his own yield function corresponding to the altitude above the sea level.

The computation of NM rigidity cut-off and asymptotic directions is performed with the MAGNETOCOSMICS code [18] using the combination of IGRF (epoch 2015) as the internal field and the Tsyganenko 89 as external field [19]. This combination provides balance between simplicity and realism [20,21]. For the galactic cosmic ray (GCR) spectrum we apply the force-field model $[22,23]$ with a solar modulation parameter calculated similarly to [24]. The optimization is performed using the Levenberg-Marquardt method $[25,26]$ by minimization of the difference between the modelled and measured NM responses i.e.optimization of the functional $\mathscr{F}$ over the vector of $n$ unknowns and $m$ NM stations:

$$
\mathscr{F}=\sum_{i=1}^{m}\left[\left(\frac{\Delta N_{i}}{N_{i}}\right)_{\text {mod. }}-\left(\frac{\Delta N_{i}}{N_{i}}\right)_{\text {meas. }}\right]^{2}
$$

In our model we can assume different spectral shapes, namely a modified power law with variable slope rigidity spectrum of SEPs similarly to [10,27]:

$$
J_{\|}(P)=J_{0} P^{-(\gamma+\delta \gamma(P-1))}
$$

where $J_{\|}(P)$ is the particle flux with given rigidity $P$ arriving from the Sun along the axis of symmetry whose direction is defined by geographic coordinate angles $\Psi$ and $\Lambda$ (latitude and longitude), $\gamma$ is the power-law spectral exponent at rigidity $\mathrm{P}=1 \mathrm{GV}, \delta \gamma$ is the rate of the spectrum steepening. We can assume also an exponential spectrum similarly to [27]:

$$
J_{\|}(P)=J_{0} \exp \left(-P / P_{0}\right)
$$

where $J_{\|}$is defined as in Eq. (2.3) and $P_{0}$ is a characteristic proton rigidity.

Accordingly, the pitch angle distribution in both cases is assumed to be a superposition of two Gaussians, which allows us to model a bidirectional particle flow:

$$
G(\alpha(P)) \sim \exp \left(-\alpha^{2} / \sigma_{1}^{2}\right)+B * \exp \left(-(\alpha-\pi)^{2} / \sigma_{2}^{2}\right)
$$

where $\alpha$ is the pitch angle, $\sigma_{1}$ and $\sigma_{2}$ are parameters describing the width of the pitch angle distribution, $\mathrm{B}$ is a parameter corresponding to the contribution of the particle flux arriving from the anti-sun direction. Therefore, according to equations (2.3) to (2.5) eight (seven) parameters have to be determined: $J_{0}, \gamma, \delta \gamma, \Psi, \Lambda, \sigma_{1}, \sigma_{2}, B$ in the case of modified power-law rigidity spectrum (Eq. 2.3) or $J_{0}, P_{0}, \Psi, \Lambda, \sigma_{1}, \sigma_{2}, B$ in the case of exponential spectrum (Eq.2.4). 


\section{Derived spectral and angular characteristics of SEP}

An example of the application of the method is the analysis of the third Halloween event, namely GLE 67 on 2 November 2003. The event is related to X8.3/2B solar flare with onset at about 17:30 - 17:35 UT. The strongest NM increases were observed at South Pole (36.0\%), McMurdo (15.2\%), Terre Adelie (14.2\%) and Fort Smith (12.0\%) compared to pre-increase levels. The event was characterized by a large anisotropy in its initial phase, since no significant increase at SANAE NM was observed. Several computed NM asymptotic cones are shown in Fig.1. The derived rigidity spectra using (Eq.2.3) with the corresponding PAD are presented in Fig.2. During the event's onset, SEPs had relatively hard rigidity spectrum and strong anisotropy as it was observed by NM stations with small pitch-angles, specifically South Pole, Terre Adelie and McMurdo. In addition, there is a clear indication for a bidirectional particle flux (Eq.2.5), the details are given elsewhere [28]. The contour plots of residuals for the best fit solutions vs. geographic latitude and longitude during the main phase of the event (18:00 UT) are presented in Fig.4a. One can see that the SEP rigidity spectrum gradually soften throughout the event. After 18:10 UT the derived rigidity spectra are described by a pure power law.

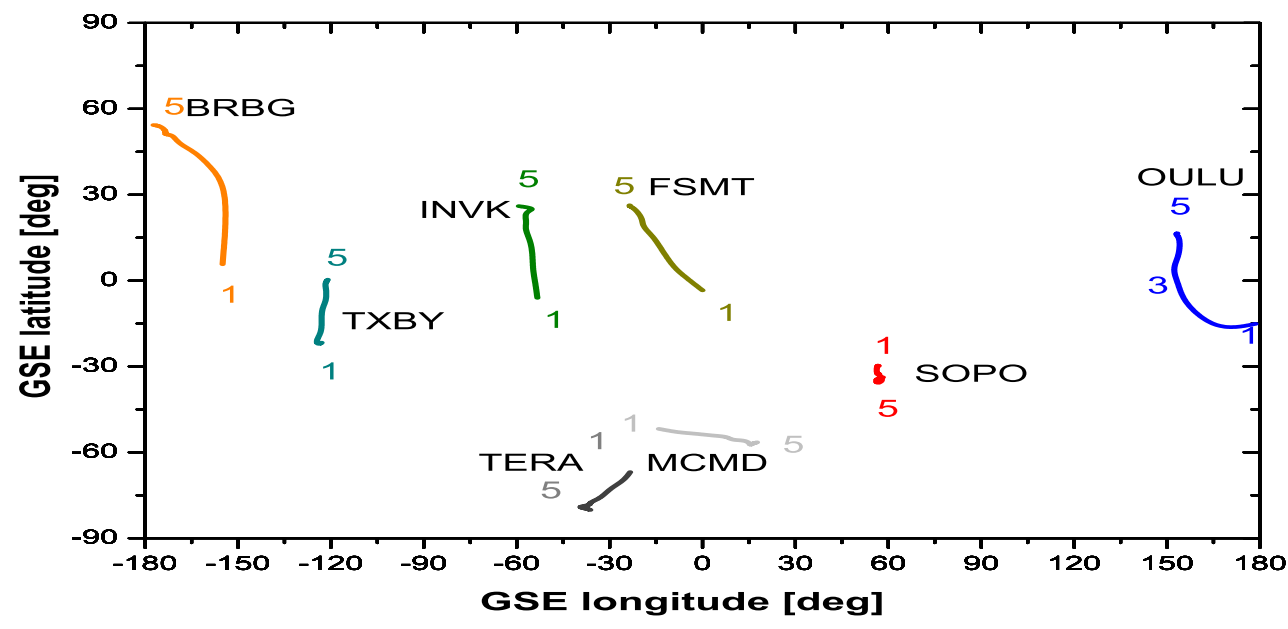

Figure 1: Asymptotic viewing cones at different NM stations on the onset of 2 November 2003 at 17:30 UT in the GSE coordinates. The abbreviations, the color lines and the numbers, indicate the NM stations, the proton arrival directions and the rigidities of arriving protons in GV.

The second event presented in this study occurred during the declining phase of the solar cycle. On 13 December 2006, NOAA active region 10930, produced X3.4/4 B solar flare associated with full-halo CME accompanied by a SEP event [29, 30]. The event was recorded by several NMs with maximum seen at Oulu NM $\sim 90 \%$ (5 min data) and was classified as GLE 70. The derived rigidity spectra and PADs are presented in Fig.3. In this case there is no evidence of SEPs arriving from anti-Sun direction. At the event's onset, SEPs possess hard spectrum and strong anisotropy as a beam like flux observed by NM stations with small pitch-angles. The SEP spectra soften throughout the event and the angular distribution broadened out after the initial phase. Details of the derived spectral and angular characteristics are given in [31]. The contour plots of residuals for 

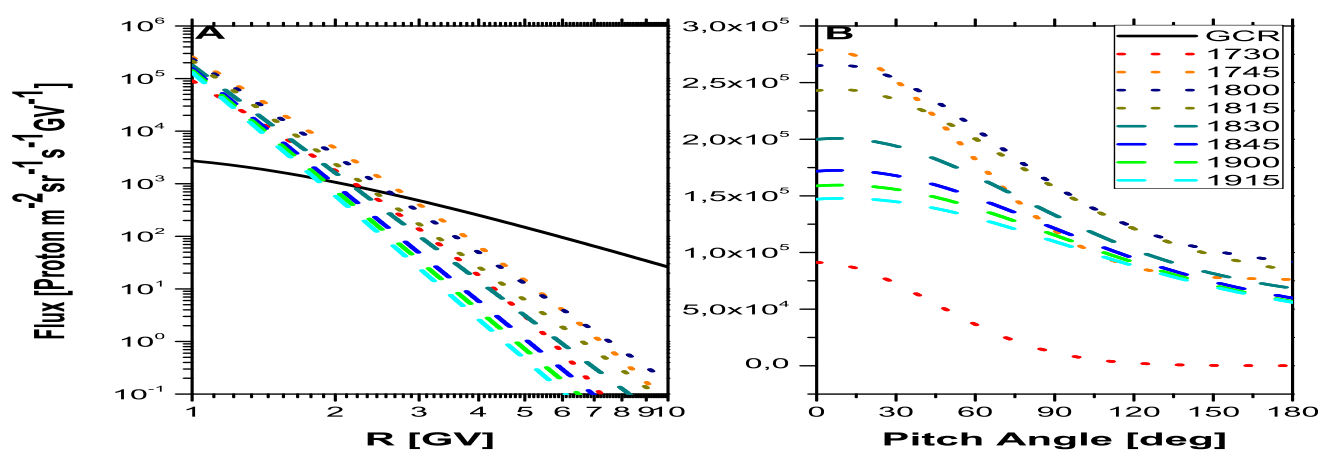

Figure 2: Derived rigidity spectra and pitch angle distributions of GLE particles during 2 November 2003 event. Time refers to the beginning of the corresponding 5-minute interval as denoted in the legend. The solid black line in the left panel depicts GCRs.

the best fit solutions vs. geographic latitude and longitude during the late phase of the event (06:00 UT) are presented in Fig.4b and good agreement with derived apparent source position is achieved (for details see Table 2 in [31]).
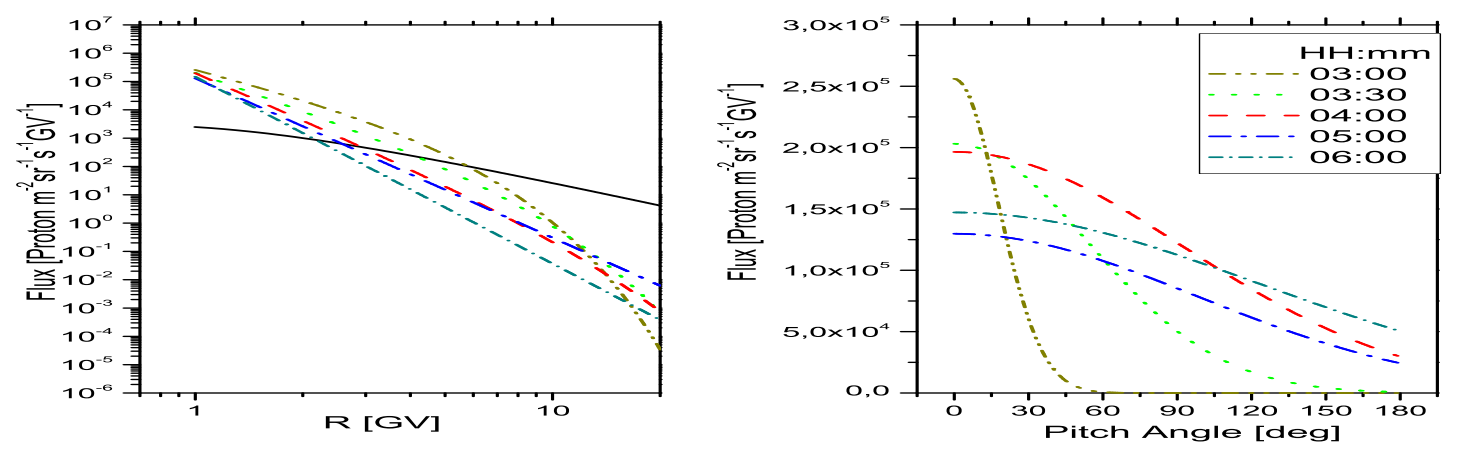

Figure 3: Derived rigidity spectra and pitch angle distributions of GLE particles during GLE 70 event on 13 December 2006. Time refers to the beginning of the corresponding 5-minute interval as denoted in the legend. The solid black line in the left panel depicts GCRs.

The described method is applied for analysis of sub-GLE events. After the start of operation of DOM C/B NMs [32], the worldwide NM network became more sensitive to the registration of SEP events, because high elevation polar NMs possess lower compared to the sea level atmospheric cut-off. As a result, the high altitude polar NM stations are able to detect SEP events, which would not be registered by the other (nearly sea level) NMs (threshold energy of about $300 \mathrm{MeV}$ instead of $450 \mathrm{MeV}$ ). Such event would be classified as sub-GLE (details given in this volume). In order to perform a consistent convergence of the optimization we simplify the model by assuming a pure power law rigidity spectrum and Gaussian PAD without bidirectional particle flux and assume the apparent source position along the IMF. Hence we reduce the number of unknowns to be found to three. However, as a result of the optimization we derive several solutions with similar quality as residual [33]. Subsequently with this new set of parameters we perform full Monte Carlo simulation 


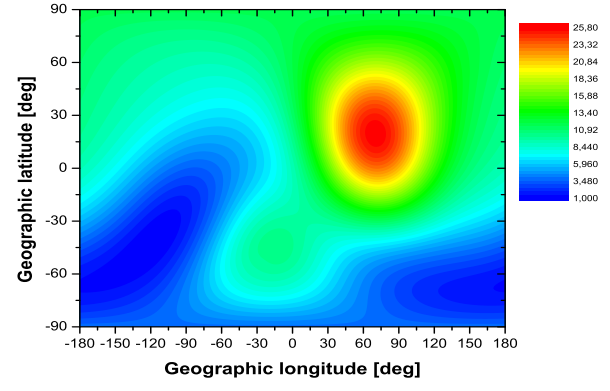

(a) GLE 67 on 2 November 2003

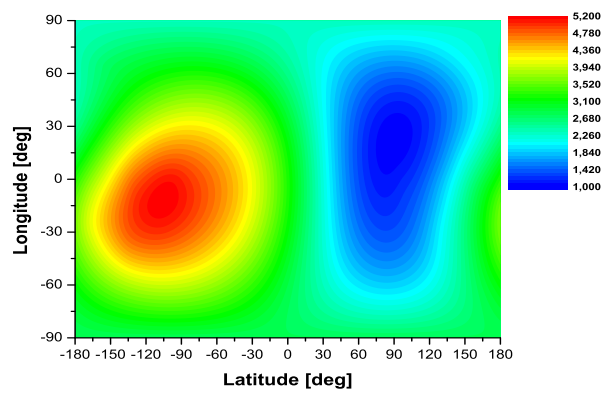

(b) GLE 70 event on 13 December 2006

Figure 4: Contour plot of sum of variances for the best fit solutions vs. geographic latitude and longitude.

of the global NM network response by fixing the PAD and spectral characteristics, but varying the apparent source position location. The best fit of global NM network response of this forward modelling is assumed as the final apparent source position (the croos in Fig.5c), subsequently used as input for the next iteration of the optimization procedure. An example is presented on Fig. 5a,b (assessed spectra and PAD of sub-GLE event on 29 October 2015).

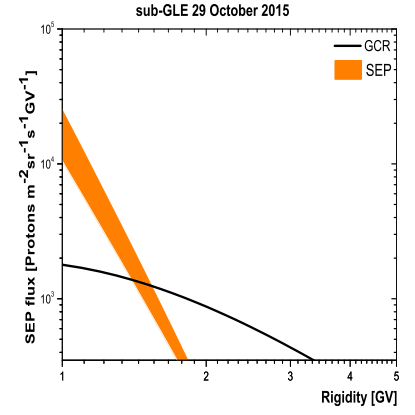

(a) Derived family of rigidity spectra (orange pattern)

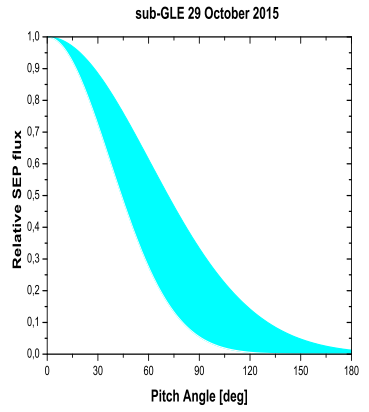

(b) Derived family of PADs (light blue pattern)

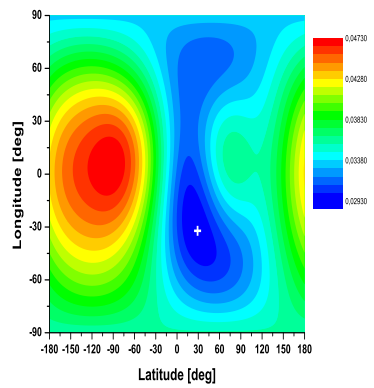

(c) Contour plot for the best fit solutions.

Figure 5: sub-GLE on 19 October 2015. The cross in panel C depicts the apparent source position.

Table 1: List of GLE events studied with our model. The rows depicts the number and date of the event, the status i.e. the time interval within derived spectral and angular characteristics and the corresponding reference for details.

\begin{tabular}{|c|c|c|c|c|c|c|c|c|}
\hline GLE & GLE 59 & GLE 60 & GLE 65 & GLE 66 & GLE 67 & GLE 69 & GLE 70 & GLE 71 \\
\hline Date & 14.07 .2000 & 15.04 .2001 & 28.10 .2003 & 29.10 .2003 & 02.11 .2003 & 20.01 .2005 & 13.12 .200670 & 017.05 .2012 \\
\hline $\begin{array}{c}\text { Status } \\
\text { Reference }\end{array}$ & $\begin{array}{c}5 \mathrm{~min} \\
{[31]}\end{array}$ & $\begin{array}{l}\text { in progress } \\
\text { SEP server }\end{array}$ & $\begin{array}{l}\text { preliminary } \\
\text { ECRS } 2016\end{array}$ & $\begin{array}{l}\text { preliminary } \\
\text { ECRS } 2016\end{array}$ & $\begin{array}{c}5 \text { min } \\
{[28]}\end{array}$ & in progress & $\begin{array}{c}5 \min \\
{[31]}\end{array}$ & $\begin{array}{c}5 \mathrm{~min} \\
{[15]}\end{array}$ \\
\hline
\end{tabular}




\section{Conclusion}

Here, we presented method for GLE analysis using the global NM data. The method was successfully applied for analysis of GLEs without features (GLE 59 and GLE 70), with particles arriving from anti-sunward direction (GLE 67) and sub-GLEs.The full list of our analysed GLE events is given in Table 1. This work was supported by the Center of Excellence ReSoLVE (project No. 272157) of the Academy of Finland. We acknowledge the NMDB and PIs of NM stations used in these studies.

\section{References}

[1] H. Debrunner, E. Flückiger, H. Gradel, J. Lockwood, and R. McGuire, Observations related to the acceleration, injection, and interplanetary propagation of energetic protons during the solar cosmic ray event on February 16, 1984, Journal of Geophysical Research 93 (1988), no. A7 7206-7216.

[2] J. A. Lockwood, H. Debrunner, and E. O. Flükiger, Indications for diffusive coronal shock acceleration of protons in selected solar cosmic ray events, Journal of Geophysical Research: Space Physics 95 (1990), no. A4 4187-4201.

[3] D. Reames, Particle acceleration at the sun and in the heliosphere, Space Science Reviews 90 (1999), 413-491.

[4] E. Cliver, S. Kahler, and D. Reames, Coronal shocks and solar energetic proton events, Astrophysical Journal 605 (2004) 902-910.

[5] D. Reames, The two sources of solar energetic particles, Space Sci. Rev. 175 (2013), 53-92.

[6] M. Desai and J. Giacalone, Large gradual solar energetic particle events, Living Reviews in Solar Physics 13 (2016), no. 13.

[7] M. Shea and D. Smart, A summary of major solar proton events, Solar Physics 127 (1990) 297-320.

[8] M. Shea and D. Smart, Possible evidence for a rigidity-dependent release of relativistic protons from the solar corona, Space Science Reviews 32 (1982) 251-271.

[9] J. Humble, M. Duldig, D. Smart, and M. Shea, Detection of 0.5-15 GeV solar protons on 29 September 1989 at Australian stations, Geophysical Research Letters 18 (1991), no. 4 737-740.

[10] J. Cramp, M. Duldig, E. Flückiger, J. Humble, M. Shea, and D. Smart, The October 22, 1989, solar cosmic enhancement: ray an analysis the anisotropy spectral characteristics, Journal of Geophysical Research 102 (1997), no. A11 24 237-24 248.

[11] E. Vashenyuk, Y. Balabin, J. Perez-Peraza, A. Gallegos-Cruz, and L. Miroshnichenko, Some features of the sources of relativistic particles at the sun in the solar cycles 21-23, Advances Space Research 38 (2006), no. 3 411-417.

[12] H. Mavromichalaki, A. Papaioannou, C. Plainaki et al., Applications and usage of the real-time neutron monitor database, Advances of Space Research 47 (2011) 2210-2222.

[13] J. Bieber and P. Evenson, Spaceship earth - an optimized network of neutron monitors, in Proc. of 24th ICRC Rome, Italy, 28 August - 8 September 1995, vol. 4, pp. 1316-1319, 1995.

[14] R. Bütikofer and E. Flückiger, Differences in published characteristics of GLE 60 and their consequences on computed radiation dose rates along selected flight paths, Journal of Physics: Conference Series 409 (2013), no. 1012166. 
[15] A. Mishev, L. Kocharov, and I. Usoskin, Analysis of the ground level enhancement on 17 May 2012 using data from the global neutron monitor network, J.of Geophysical Res. 119 (2014) 670-679.

[16] A. Mishev, I. Usoskin, and G. Kovaltsov, Neutron monitor yield function: New improved computations, Journal of Geophysical Research 118 (2013) 2783-2788.

[17] A. Gil, I. Usoskin, G. Kovaltsov, A. Mishev, C. Corti, and V. Bindi, Can we properly model the neutron monitor count rate?, J. Geophys. Res. 120 (2015) 7172-7178.

[18] L. Desorgher, E. Flückiger, M. Gurtner, M. Moser, and R. Bütikofer, A GEANT 4 code for computing the interaction of cosmic rays with the earth's atmosphere, International Journal of Modern Physics A 20 (2005), no. A11 6802-6804.

[19] N. Tsyganenko, A magnetospheric magnetic field model with a warped tail current sheet, Planetary and Space Science 37 (1989), no. 1 5-20.

[20] K. Kudela and I. Usoskin, On magnetospheric transmissivity of cosmic rays, Czechoslovak Journal of Physics 54 (2004), no. 2 239-254.

[21] J. Nevalainen, I. Usoskin, and A. Mishev, Eccentric dipole approximation of the geomagnetic field: Application to cosmic ray computations, Advances in Space Research 52 (2013), no. $122-29$.

[22] L. Gleeson and W. Axford, Solar modulation of galactic cosmic rays, Astrophysical Journal 154 (1968) 1011-1026.

[23] R. Caballero-Lopez and H. Moraal, Limitations of the force field equation to describe cosmic ray modulation, Journal of Geophysical Research 109 (2004) A01101.

[24] I. Usoskin, G. Bazilevskaya, and G. Kovaltsov, Solar modulation parameter for cosmic rays since 1936 reconstructed from ground-based neutron monitors and ionization chambers, Journal of Geophysical Research 116 (2011) A02104.

[25] K. Levenberg, A method for the solution of certain non-linear problems in least squares, Quarterly of Applied Mathematics 2 (1944) 164-168.

[26] D. Marquardt, An algorithm for least-squares estimation of nonlinear parameters, SIAM Journal on Applied Mathematics 11 (1963), no. 2 431-441.

[27] E. Vashenyuk, Y. Balabin, B. Gvozdevsky, and L. Schur, Characteristics of relativistic solar cosmic rays during the event of December 13, 2006, Geomagnetism and Aeronomy 48 (2008), no. 2 149-153.

[28] L. Kocharov, S. Pohjolainen, A. Mishev et al., Investigating the origins of two extreme solar particle events: Proton source profile and associated electromagnetic emissions, The Astrophysical Journal 839 (2017), no. 279.

[29] N. Gopalswamy, H. Xie, S. Yashiro, S. Akiyama, P. Mäkelä, and I. Usoskin, Properties of ground level enhancement events and the associated solar eruptions during solar cycle 23, Space Science Reviews 171 (2012), no. 1-4 23-60.

[30] H. Moraal and K. McCracken, The time structure of ground level enhancements in solar cycle 23, Space Science Reviews 171 (2012), no. 1-4 85-95.

[31] A. Mishev and I. Usoskin, Analysis of the ground level enhancements on 14 July 2000 and on 13 December 2006 using neutron monitor data, Solar Physics 291 (2016), no. 4 1225-1239.

[32] S. Poluianov, I. Usoskin, A. Mishev, H. Moraal, H. Krüger, G. Casasanta, R. Traversi, and R. Udisti, Mini neutron monitors at Concordia research station, central Antarctica, Journal of Astronomy and Space Science 32 (2015), no. 4 281-287.

[33] D. Himmelblau, Applied Nonlinear Programming. Mcgraw-Hill(Tx), 1972. 